\title{
Regularization and Adaptation for the Approximation of Nonsmooth Solutions for Fredgolm First Kind Integral Equations
}

\author{
T.I. Serezhnikova \\ Institute of Mathematics and Mechanics UrB RAS, \\ Ural Federal University,Ekaterinburg, Russia \\ *Corresponding Author: sti@imm.uran.ru
}

Copyright $\odot 2014$ Horizon Research Publishing All rights reserved.

\begin{abstract}
In the paper, we propose a new regularization algorithm based on the generalized Tikhonov regularization method.

In the paper proposed technique treats problems in the form of Fredholm first kind integral equations which must be inverted.

In classical regularization functionals we propose put in the specialize summand, which allows to distribution control of points of approximate solutions.

The best additional summand one can get with using more information about solutions.

There are six model numerical results in the paper. Numerical experiments prove, that our technique does a better job of preserving functions jumps.

As result in the case of the image reconstructions problems, we obtain approximate solutions of better accuracy and images become more blur-free images.
\end{abstract}

Keywords Image Reconstruction; Nonsmooth Solutions; Ill-posed Problems; Tikhonov Regularization; Integral Equations; Adaptive Method

\section{Introduction}

The field of inverse problems is of large interest due to the importance of applications to science and industry. Since most inverse problems cannot be solved analytically, computational methods play a fundamental role. There are many techniques to treat inverse problems formulated in the form of Fredholm first kind integral equations which must be inverted.

Inverse problems typically involve the quantities based on indirect measurements, so that noise in the data can cause significant errors in computational results. Techniques known as regularization methods have been developed to deal with this ill-posedness.

In the paper, we describe two new regularizing algorithms for numerical reconstructing nonsmooth solutions of the Fredholm first kind integral equations.
These two algorithms are based on generalized Tikhonov regularization with non-differentiable functionals.

The main goal of our research is to develop numerical techniques (based on Tikhonov regularization methods) which provide efficient approximations of nonsmooth solutions for Fredholm first kind integral equations.

The problem of the reconstruction of blurred and noisy images is a source of many interesting models.

In the problem of the reconstruction of images, objects (pre-images) are specified by two-dimensional functions. For these functions, many plane cutting lines may be kinked functions, may have points of the function discontinue, points of function jumps. The main goal of our numerical experiments is to construct algorithms which provide better approximations of such functions. We propose the special summand put in algorithms formulas. This summand allows to distribution control of points on such lines of approximate functions.

As result, we obtain approximate solutions of better accuracy and reconstructed images become more accurate. Of cause, the best additional summand we can get with using more information about solutions.

We use iterative technique known as the subgradient method providing efficient means of solving linear systems, which are large and ill-conditioned.

In the paper, we describe numerical results and demonstrate figures for six models. We emphasize two models for the atmospheric optics deblurring problem.

One can examine numerical results and compare how our technique does a better job of preserving functions jumps in both one-dimensional models and twodimensional ones.

We want to demonstrate our figures of models and to inform the scientific community about our numerical results. We do not intend to carry out any proofs in this paper. One can see that our technique allows researchers to compute image reconstructions and to get more blurfree images.

The paper is organized as follows. In the next section, we will define regularization functionals and will present the based constructions for two techniques, which will be used further. In Sections 3 and 4, we describe the com- 
putational problems that must be solved in practice. In Subsection 5.1, we write an adapting form for the specialize summand. In Subsection 5.2, one can examine graphic results to compare two techniques for image deblurring reconstructions. Section 6 contains conclusions and acknowledgements.

\section{Problem Formulation}

In this section we consider two regularization techniques for linear operator equations. Let $A: U \rightarrow F$ be a linear operator, and let $U$ and $F$ be linear normed spaces. Assume that the inverse operator $A^{-1}$ is discontinuous, then the equation $A u=f$ is said to be ill-posed problem.

Abstract methods with full investigation convergence of two regularization algorithms for this problem presented in [1-4].

Let $f_{\delta}$ be inaccurate data to $f,\left\|f-f_{\delta}\right\| \leq \delta$. The foundation of the first regularization method is given by

$$
\min \left\{\left\|A u-f_{\delta}\right\|_{C[\Pi]}+\alpha\|u\|_{H^{\mu}}: u \in H^{\mu}[\Pi]\right\}
$$

where

$$
\|u(x)\|_{H^{\mu}}=\max _{x \in \Pi}|u(x)|+\sup _{x_{1}, x_{2} \in \Pi} \frac{\left|u\left(x_{1}\right)-u\left(x_{2}\right)\right|}{\left|x_{1}-x_{2}\right|^{\mu}}
$$

$H^{\mu}=H^{\mu}[\Pi]$ presents the norm in the Lipschitz space of functions, and $\Pi$ is a compact set.

The foundation of the second regularization method is given by

$$
\min \left\{\left\|A_{h} u-f_{\delta}\right\|_{L_{2}}^{2}+\alpha\left(\|u\|_{L_{2}}^{2}+J(u)\right): u \in U\right\}
$$

here

$$
J(u)=\int_{D}|\nabla u| d x
$$

where $\nabla u$ denotes the gradient of smooth function $u,\left(u \in W_{1}^{1}(D)\right), J(u)$ is the total variation of the function $u$ on $D$.

Usually, in regularization methods the proper regularization parameter $\alpha$ will give an approximation solution, which may be overly smooth. Our goal is to reconstruct nonsmooth solutions.

We propose to combine two process: the Tikhonov variational representations (1)-(4) and the version of the iterative technique, see [5-7]. Then, the result procedure generates a sequence $\left\{u^{k}\right\}$ by taking $u^{k}$ to be the minimizer

$$
\begin{array}{r}
u^{k}=\arg \min \left\{\Phi^{\alpha}(u)+\beta\left\|u-u^{k-1}\right\|^{2}: u \in U\right\} \\
\equiv \arg \min \left\{\Phi^{\alpha, \beta}\left(u ; u^{k-1}\right)\right\}, \beta>0,
\end{array}
$$

where $\Phi^{\alpha}(u)$ is functional in (1) or in (3), $\|\cdot\|$ denotes Hilbert norm.

In order to compute $u^{k}$ defined in (5), we use iterative nonlinear subgradient method

$$
u^{k-1, \nu+1}=u^{k-1, \nu}-\lambda_{k-1} \frac{v^{k-1, \nu}}{\left\|v^{k-1, \nu}\right\|}, \quad \nu=0,1,2, \ldots, n_{k-1},
$$

where $v^{k-1, \nu} \in \partial \Phi^{\alpha, \beta}\left(u^{k-1, \nu}\right), \quad \Phi^{\alpha, \beta}$ is functional in (5), and $\partial \Phi$ is an arbitary subgradient of the functional $\Phi$.

Stability of process (6) is guaranteed provided that the functional in (5) is strong convex, see [3].

\section{Numerical Experiments in One-Dimensional Space}

First of all, we tested the regularization method (1),(2),(5),(6) for the one-dimensional problem.

Consider the Fredholm first kind integral equation

$$
\begin{aligned}
A u \equiv \int_{0}^{2} \frac{H}{H^{2}+(x-y)^{2}} u(x) d x & =f(y), \\
& 0<H \leq 2,0 \leq x, y \leq 2 .
\end{aligned}
$$

The inverse problem associated with the model equation (7) is the following. Given measurements $f(y)$ on the earth's surface, reconstruct the subsurface gravitation field $u(x)$. The parameter $H$ represents the depth.

We are interested in reconstructions of nonsmooth solutions. The model functions $u_{\text {true }}$ were taken to be piecewise linear.

We selected the proper constant $\beta$ in (5) for each numerical experiments.

Our numerical experiments proved that it is useful to change $\beta$ if we have changed amount of mash points; see details in [3]. The initial guess was taken to be a zero vector $u^{0}=0$ in (5).

We used the relative iterative solution error norms to measure numerical performance in tests

$$
\Delta_{1}=\frac{\left\|u_{\text {true }}-\tilde{u}_{\alpha}\right\|_{L_{2}}}{\left\|u_{\text {true }}\right\|_{L_{2}}}, \quad \Delta_{2}=\frac{\left\|A \tilde{u}_{\alpha}-f\right\|_{L_{2}}}{\|f\|_{L_{2}}}
$$

where $u_{\text {true }}$ is the true solution of the functional equation in (7) and $\tilde{u}_{\alpha}$ represents the numerical approximation to $u_{\text {true }}$. One can see solution error quantities and other details in the paper [3].

In this paper we only present nine plots of numerical test results for the one-dimensional problem (7); see Figure 1.

One can see in Figure 1 that our technique, (1), (2) together with (5),(6), does a good job of preserving local extreme points and jumps of functions. 


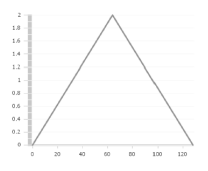

(a)

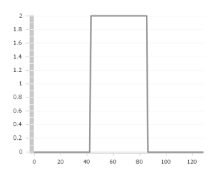

(d)

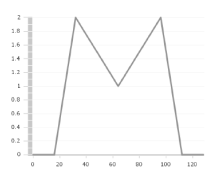

(g)

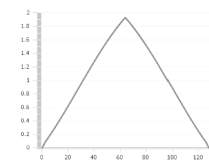

(b)

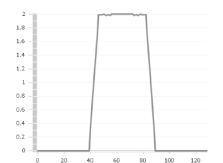

(e)

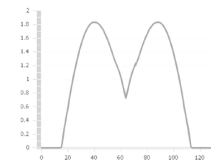

(h)

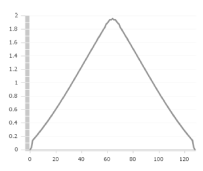

(c)

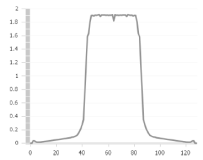

(f)

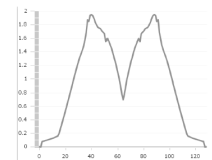

(i)
Figure 1. Models and numerical solutions. (Mesh points: $x_{i}=$ $i h, i=0, \ldots, 128, h=1 / n, n=128)$. Plots in the first column show three true solutions. Plots in the second column represent reconstructions in the case of the numerical exact $f$ in (7). The third column consists of reconstructions $u$ in the case of noisy data $f$ in (7).

\section{Mathematicall Model for Im- age Deblurring}

We consider the follwing two-dimensional Fredgolm first kind integral equation:

$$
A u \equiv \int_{0}^{1} \int_{0}^{1} K(x-\xi, y-\eta) u(x, y) d x, d y=f(\xi, \eta)
$$

In image reconstruction, the estimation of $u$ from observation of $f$ is referred to as the two-dimensional image deblurring problem.

In optics, $u$ is called the light source, or object. The kernel function $K$ is known as the point spread function (PSF), and $f$ is called the blurred image. The image is often recorded with a device known as a CCD camera.

We are interested in reconstructions of nonsmooth solutions. Using total variation, one can effectively reconstruct functions with jump discontinuities.

We construct the original method (see $(3),(4)$ together with $(5),(6))$ to solve problem (8). The practical implementation of this method requires the minimization of a discretized version of the functional in (8). A completely discrete model may be obtained by truncating the region of integreation in (8) to be the union of the small squares $h \times h, h=1 . / n$ and then applying the midpoint quadrature to (8). So, equations (3)-(6) are reduced to

$$
\begin{aligned}
& \min \left\{\sum_{k, l} h^{2}\left[\sum_{i, j} h^{2} K\left(y_{k}-t_{i}, y_{l}-s_{j}\right) u\left(t_{i}, s_{j}\right)-f_{k, l}\right]^{2}\right. \\
& \left.+\alpha \sum_{i, j} h^{2}\left\{u_{i, j}^{2}+\left[\left(\frac{u_{i, j}-u_{i, j-1}}{h}\right)^{2}+\left(\frac{u_{i, j}-u_{i-1, j}}{h}\right)^{2}\right]^{\frac{1}{2}}\right\}\right\},
\end{aligned}
$$

$$
\mathbf{u}^{k}=\arg \min \left\{\Phi_{N}^{\alpha}(\mathbf{u})+\sum_{i, j} \beta_{i, j}\left(u_{i, j}-u_{i, j}^{k-1}\right)^{2}: \mathbf{u} \in R^{N}\right\}
$$

Here, $N=n^{2}, \Phi_{N}^{\alpha}(\mathbf{u})$ is functional in (9).

We use the iterative subgradient method in order to compute $\mathbf{u}^{k}$ defined in (10)

$$
\mathbf{u}^{k, \nu+1}=\mathbf{u}^{k, \nu}-\lambda_{k} \frac{\mathbf{v}^{k, \nu}}{\left\|\mathbf{v}^{k, \nu}\right\|}, \quad \nu=0,1,2, \ldots, n_{k},
$$

where $\mathbf{v}^{k, \nu} \in \partial \Phi_{N}^{\alpha, \beta}\left(\mathbf{u}^{k, \nu}\right)$ is the functional in (10), and $\partial \Phi$ is an arbitary subgradient of the functional $\Phi$.

\section{Adaptive Function $\beta(x, y)$}

\subsection{Motivation}

For the one-dimensional problem, we used constant parameter $\beta$ in every points for the same discrete model. It may be useful to change parameter $\beta$ if we have changed amount of mash points, see details in [3].

The first model for the two-dimensional problem (see Figure 2(a)) is resembling the second model, see Figure 1(d), for the one-dimensional problem. Namely, failure of the initial calculations for the model in Figure 2 motivated us to change the way of $\beta_{i, j}$ selection. Now, we propose to use an adapted $\beta_{i, j}$. We set

$\left\{\beta_{i, j}=10^{-10}\right.$ for the points, where $u_{\text {true }}=\max \left\{u_{\text {true }}\right\}$, $\left\{\beta_{i, j}=0\right.$, where $u_{\text {true }}<\max \left\{u_{\text {true }}\right\}$.

If the true solution $u_{\text {true }}$ is unknown, in the opinion of the author, we can make use of the approximation $\tilde{u}^{k}, k=0,1, \ldots$. But, it is outside the scope of this paper.

\subsection{Comparisons with Total Variation Regu- larization Solutions for Image Deblurring Reconstrutions}

We now compare numerical results obtained for setting a zero parameter $\beta, \beta \equiv 0$, and numerical results for adapted $\beta_{i, j}$ in (10). One can see that our numerical algorithm (3)-(6) with the parameter $\beta \equiv 0$ is a variant of the Tikhonov total variation regularization technique in two-dimensional case. In order to demonstrate the qualitative difference between numerical results obtained for setting $\beta \equiv 0$ and for setting adapted $\beta_{i, j}$, we present plots of two blurred image reconstructions in this subsection. It is very interesting to compare the performance of these two solution techniques. An overall cost comparison is difficult to carry out, since it depends on many 


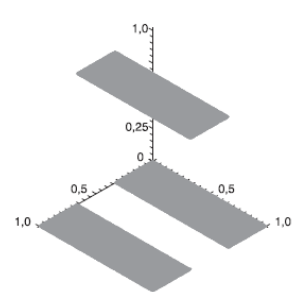

(a)

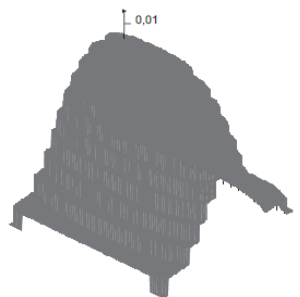

(b)

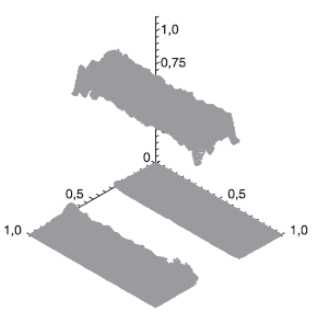

(c)

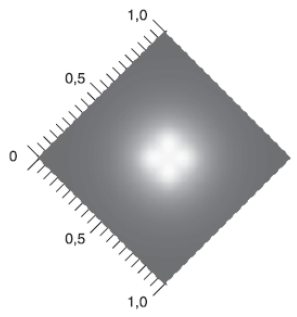

(a)

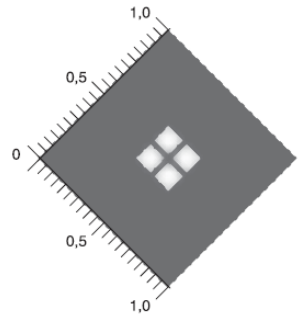

(b)

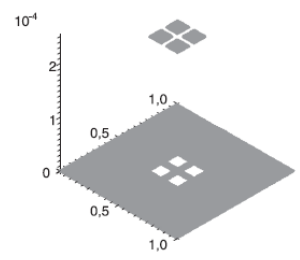

(c)

Figure 2. First model image reconstruction. (a) Plot shows the true object. (b) Plot shows blurred image. (c) Plot shows reconstrution from data (b).

factors. Our numerical results produce visual comparisons of these two solution techniques for two tests, see Figure 3 and Figure 5.

In order to compare the results, we present the plot of the true image diagonal and plots of the corresponding reconstruction diagonals in Figure 4.

For the solution of equation (8), the diagonal $u_{\text {true }}(x, x)$ of the true image, see Figure $3(\mathrm{c})$, is given by

$$
u_{\text {true }}(x, x)=\left\{\begin{array}{cc}
2.5 \times 10^{-4}, & 0.391 \leq x<0.484 \\
& 0.531 \leq x<0.625 \\
0 . & \text { otherwise }
\end{array}\right.
$$

In Figure 4, we have visual comparisons of two solution reconstructions for blurred image in Figure 3(a). One can see that the algorithm with adapted $\beta_{i, j}$ does a better job of preserving jumps of the function (compare Figure 4(b) with Figure 4(a)).

The base configuration for the true model in Figure 5(a) is taken from [8]. In Figure 5(a) the most interesting detail is the white triangle. Figure 6 demonstrates visual comparisons of two reconstructions for this triangle.

One can see in Figure 6(b), due to adapted $\beta_{i, j}$ the plane triangle reconstruction is better (compare with Figure $6(\mathrm{a})$ ).

Now we consider some explanation for the white triangle reconstruction in Figure 5(a). Let any fixed point $A$ lies into the white triangle and any fixed point $B$ lies out the triangle, so the point $A$ is a white point, the point $B$ is a dark point.

In our tests, for the true solution there is the point $C, C \in[A, B]$, that every point in the interval $[A, C]$ is a white point and every point in the interval $(C, B]$ is a dark point.

In our tests, for the algorithm with $\beta \equiv 0$ the result approximate solution has some intervals as $[A, B]$, which
Figure 3. Second model image reconstruction. (a) Plot shows blurred image. (b) Plot shows reconstrution from data (a) for adapted $\beta_{i, j}$ (see (12)). (c) Plot shows the true object in the threedimensional coordinate system.

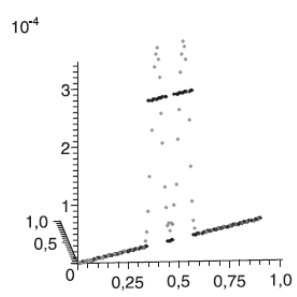

(a)

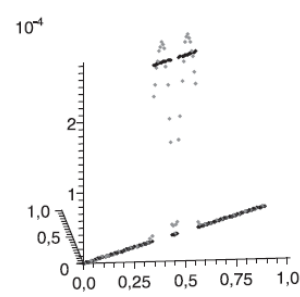

(b)
Figure 4. A plot of the true image diagonal (13) and reconstruction diagonals. (a) Plots show the true image diagonal and the reconstruction diagonal for $\beta \equiv 0$. (b) Plots show the true image diagonal and the diagonal reconstructed with the adapted $\beta_{i, j}$ given by (12).

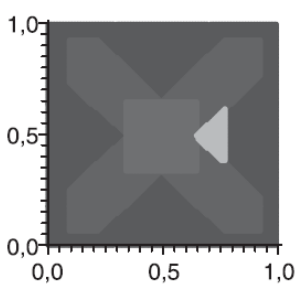

(a)

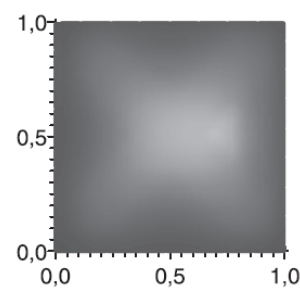

(b)

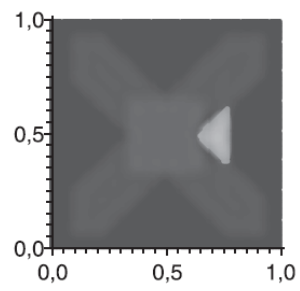

(c)

Figure 5. Plots show the $128 \times 128$ simulated satellite in Figure 5(a), the blurred image in Figure 5(b) and the reconstruction image in Figure $5(\mathrm{c})$ for adapted $\beta_{i, j}$. 
$10^{-4}$

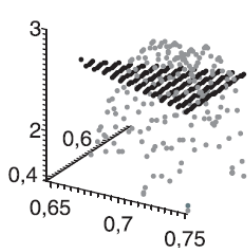

(a)
$10^{-4}$

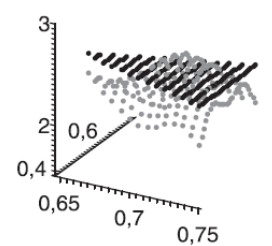

(b)
Figure 6. Plots demonstrate visual comparisons of two reconstructions for the image model in Figure 5 (a). (a) Plots show the true solution triangle part and the reconstruction of the triangle part for $\beta \equiv 0$; (b) Plots show the true solution triangle part and the reconstruction of the triangle part for adapted $\beta_{i, j}$.

not at all contain white points. So, some part of the triangle is "destroyed".

At the same time, the algorithm with adapted $\beta_{i, j}$ "save" many "almost white" points in the interval $[A, C]$.

So, the visual result is quite better in the algorithm with adapted $\beta_{i, j}$.

Additionally, let us consider finish steps of algorithms. If algorithm with $\beta \equiv 0$ is finished at the iteration number $k$ ( $k$ is number of iteration that were required to satisfy the stopping criterions), then we set the approximate solution $\tilde{\mathbf{u}}$ is equal to $\tilde{\mathbf{u}}^{k}, \tilde{\mathbf{u}}=\tilde{\mathbf{u}}^{k}$.

Then, for the algorithm with adapted $\beta_{i, j}$ we start with $\tilde{\mathbf{u}}^{k}$ as the initial approximation for iterations. Note, that due to the selection of the proper large $\beta_{i, j}$ in points of the white triangle, we have $\left.\Phi_{N}^{\alpha, \beta}\left(\mathbf{u}, \mathbf{u}^{k}\right)\right) \gg$ $\left.\Phi_{N}^{\alpha, 0}\left(\mathbf{u}, \mathbf{u}^{k}\right), \quad \mid \partial \Phi_{N}^{\alpha, \beta}\left(\mathbf{u}, \mathbf{u}^{k}\right)\right) \mid \gg 0$, where $\Phi_{N}^{\alpha, \beta}, \partial \Phi_{N}^{\alpha, \beta}$ from (10).

We continue computations with adapted $\beta_{i, j}$. Then, we get $\tilde{\mathbf{u}}^{k+1}$, which is nearer to exact solution than $\tilde{\mathbf{u}}^{k}$ : as we see in tests, number of white points in the triangle for $\tilde{\mathbf{u}}^{k+1}$ is more than number of white points in the triangle for $\tilde{\mathbf{u}}^{k}$. So the approximation of the triangle configuration is more exact for the algorithm with adapted $\beta_{i, j}$

\section{Conclusions}

In this paper we present two new regularizing algorithms for numerical reconstructing nonsmooth solutions of Fredholm first kind integral equations. We propose the specialize summand put in classical regularizing functionals containing regularization parameter $\alpha$.

Proposed summand construction uses the true solution or some information about it. We mean, for example, the information about lines consisting of points of discontinuities of the function $u_{\text {true }}$ and the information about the max value of the function $u_{\text {true }}$. In model calculations, we used specialize summand in the form which one can see in (12) for the two-dimensional case. The more general form of this summand for the twodimensional case may be given by

$$
I=\int_{Q} \beta(x, y)\left[u(x, y)-u^{k}(x, y)\right]^{2} d x d y,
$$

where

$$
\begin{gathered}
\left\{\begin{array}{l}
\beta(x, y)=u_{\max }, \quad \text { for } \quad(x, y) \in Q, \\
\beta(x, y)=0, \quad \text { for } \quad(\mathrm{x}, \mathrm{y}) \in \Pi / \mathrm{Q},
\end{array}\right. \\
u_{\max }=\max _{\Pi}\left\{u_{\text {true }}(x, y)\right\}, \Pi=[0,1] \times[0,1], \\
(x, y) \in Q \Leftrightarrow u(x, y)=u_{\max } .
\end{gathered}
$$

Above one can see that $\beta(x, y)$ is piece-wise constant function and the function $\beta(x, y)$ is depending on the function $u_{\text {true }}$. One can try to use $u^{k}(x, y)$ instead of $u_{\text {true }}$.

In the paper, we describe numerical experiments and demonstrate six Figures for models. Two of demonstrated tests are related to the atmospheric optics deblurring problem.

One can see and compare in figures that our technique does a better job of preserving functions jumps in both one-dimensional models and two-dimensional ones due to adapting of the function $\beta(x, y)$. Comparisons of reconstructions in Figure 4 and comparisons of reconstructions in Figure 6 show that the adapted function $\beta(x, y)$ ensures crucially improve of reconstructions quality for nonsmooth solutions. As result, we see in Figure 3 and Figure 5 that reconstructed images appear accurate enough images, appear more blur-free images.

There is an obvious direction of the future work. We shall try to reconstruct images which contain several complicated details. The main purpose remains to improve reconstructions of function jumps and to get more blur-free images.

\section{Acknowledgments}

The author would like to express special gratitude to Prof. V.V.Vasin, from the Institute Mathematics and Mechanics UrB RAS.

This work is supported by RFBR project no. 12-0100106.

\section{REFERENCES}

[1] V.V. Vasin. Stable approximation of nonsmooth solutions to ill-posed problems, Dokl. Math. Vol.71, No.3, 419-422, 2005.

[2] V.V. Vasin. Regularization and iterative approximation for linear ill-posed problems in the space of functions of bounded variation, Proc. Steclov Inst. Math. Suppl. Vol.12, No.1, 64-77, 2002.

[3] V.V. Vasin and T.I. Serezhnikova. A regularizing algorithm for approximation of a nonsmooth solution of Fredholm integral equations of the first kind, J. VICH. TECH., Vol.15, No.2, 15-23, 2010.

[4] V.V. Vasin and T.I. Serezhnikova. Two steps method for approximation of a nonsmooth solutions and nosy image reconstructions, J. Avt. Tel., No.2, 126-135, 2004. 
[5] J.J. Moreau. Proximite et dualite dans un espace Hilberten, Bull. Soc. Math. France, Vol. 93, No. 2, 273-299, 1965.

[6] B. Martinet. Determination approachee d'un point fixe d'ne Applications pseudo-contrctante , C.R. Acad. Sci. Paris., Vol. 274, 163-165, 1972.
[7] V.V. Vasin , A.L. Ageev. Ill-posed problems with an a priory Information, Ekaterinburg: UIF "Sience", 1993.

[8] C.R. Vogel. Computational methods for inverse problems, SIAM, Rhiladelphia, 2002. 